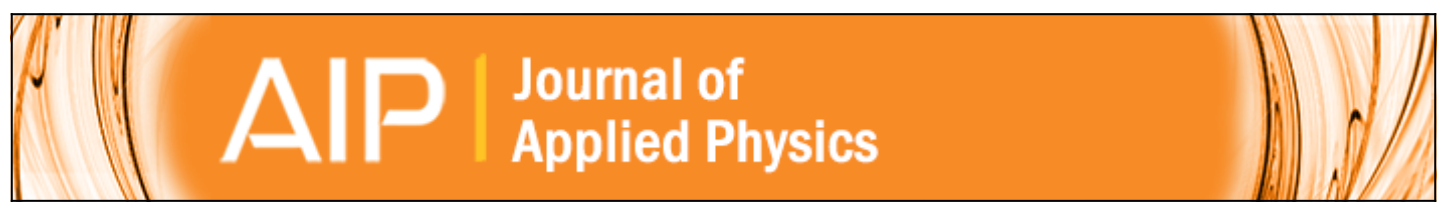

\title{
Barrier Height Studies on MetalSemiconductor Systems
}

W. G. Spitzer and C. A. Mead

Citation: Journal of Applied Physics 34, 3061 (1963); doi: 10.1063/1.1729121

View online: http://dx.doi.org/10.1063/1.1729121

View Table of Contents: http://scitation.aip.org/content/aip/journal/jap/34/10?ver=pdfcov

Published by the AIP Publishing

\section{Articles you may be interested in}

Enhancement of Schottky barrier height in heterodimensional metal-semiconductor contacts

Appl. Phys. Lett. 70, 441 (1997); 10.1063/1.118175

New method of determination of a metalsemiconductor barrier height

Appl. Phys. Lett. 25, 600 (1974); 10.1063/1.1655327

Surface States and Barrier Height of MetalSemiconductor Systems

J. Appl. Phys. 36, 3212 (1965); 10.1063/1.1702952

MetalSemiconductor BarrierHeight Measurement by the Differential Capacitance

Method-Degenerate OneCarrier System

J. Appl. Phys. 35, 3351 (1964); 10.1063/1.1713221

Metal—Semiconductor Barrier Height Measurement by the Differential Capacitance Method—One Carrier System

J. Appl. Phys. 34, 329 (1963); 10.1063/1.1702608

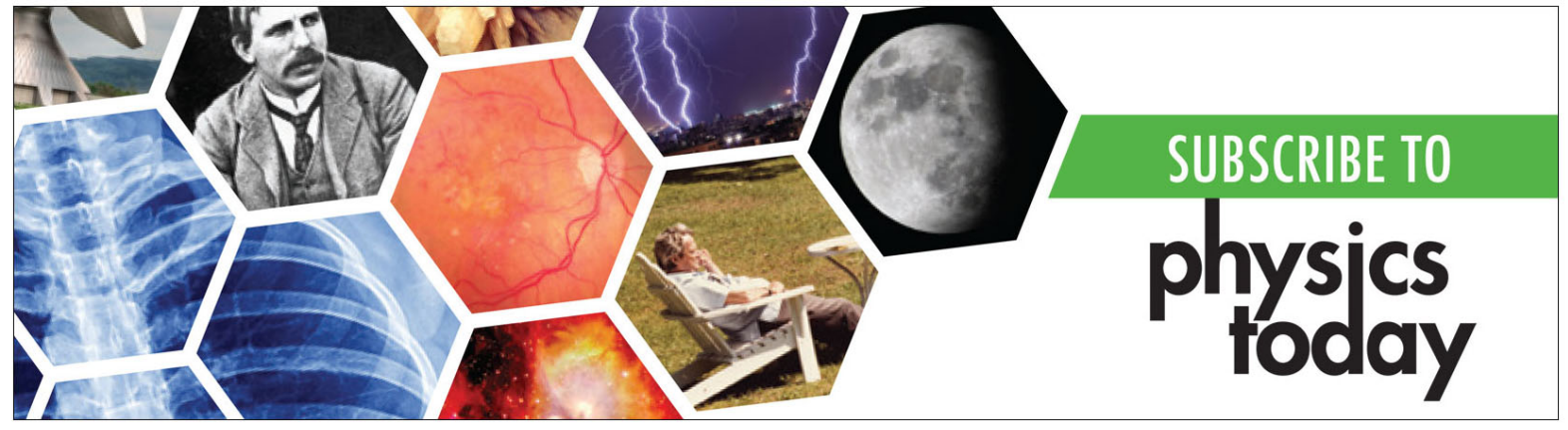




\title{
Barrier Height Studies on Metal-Semiconductor Systems
}

\author{
W. G. SPITzER* \\ Bell and Howell Research Center, Pasadena, California \\ AND \\ C. A. MEAD \\ California Insitute of Technology, Pasadena, California
}

(Received 16 April 1963; in final form 16 May 1963)

\begin{abstract}
Photovoltaic and space-charge capacitance measurements have been used to study the height of the Schottky barrier at the metal-semiconductor interface of a series of metals evaporated onto "vacuum cleaved" samples of $n$-type CdS and $n$ - and $p$-type GaAs. Although the barrier heights for metal-CdS samples increase with increasing metal work function as predicted by simple theory, significant deviations were noted. The barrier heights measured on metal-GaAs samples at different temperatures show very little dependence on the metal and appear to be fixed relative to the valence band edge by surface states. The results are compatible with the model in which the photoresponse, for photon energies less than the semiconductor energy gap, arises principally from photoemission of carriers from the metal into the semiconductor; however, the results are sensitive to the method of surface preparation and comparisons with other work are difficult.
\end{abstract}

\section{INTRODUCTION}

T HE study of the photovoltaic response of surfacebarrier rectifiers has produced considerable information on the transport of hot electrons (and holes) in metal films. In most of these studies, the system consists of a metal film deposited in some way on a semiconductor surface. In these systems, photocurrent is observed where the incident photon energy $h \nu$ exceeds the energy gap $E_{g}$ of the semiconductor. The source of this photocurrent is the band-to-band photoexcitation of carriers in the semiconductor under the metal film. It is anticipated, and has been observed experimentally, that this photocurrent is proportional to the intensity of the light transmitted by the metal film. ${ }^{1}$ However, photocurrent is also observed in many cases for $h \nu<E_{g}$. The mechanisms responsible for this photocurrent could be excitation from defect levels in the semiconductor, localized states close to the metal-semiconductor interface, or conduction electrons in the metal which have sufficient energy to surmount the potential barrier at the interface. Much of the recent work has been done with a view towards establishing photoemission from the metal film as an operating mechanism. In a few cases, studies of the spectral photoresponse with different metals for $h \nu<E_{g}$ and the dependence of this response upon the thickness of the metal film have given information on the attenuation lengths of hot electrons ${ }^{1,2}$ or holes ${ }^{3}$ of approximately $1 \mathrm{eV}$ excess kinetic energy.

In addition to the range of the hot carriers, a second parameter of interest in the photoemission process is the height of the potential or Schottky barrier and its

\footnotetext{
* Present address : Electrical Engineering Department, University of Southern California, Los Angeles, California.

${ }^{1}$ C. R. Crowell, W. G. Spitzer, L. E. Howarth, and E. E. LaBate, Phys. Rev. 127, 2006 (1962).

${ }^{2}$ W. G. Spitzer, C. R. Crowell, and M. M. Atalla, Phys. Rev. Letters 8, 57 (1962).

${ }^{3}$ C. R. Crowell, W. G. Spitzer, and H. G. White, Appl. Phys. Letters 1, 3 (1962).
}

dependence on the work function of the metal film $\varphi_{M}$, the electron affinity of the semiconductor $\chi$, and the concentration and distribution of surface states at the interface. There is some information available concerning barrier heights for different metals and semiconductors. ${ }^{1-8}$ In most cases, however, the papers are concerned with only one or two metals and one semiconductor. Any attempt to compare the work of different investigators is difficult since different methods of both semiconductor surface preparation and metal film deposition have been employed. At the present time, the only detailed study of barrier heights known to the authors is the work of Archer and Atalla ${ }^{6}$ for a series of metals on silicon. The silicon surface was prepared in a vacuum chamber by cleavage and the metal film deposited by evaporation. In a number of cases, deliberate exposure of the cleaved surface to oxygen prior to evaporation of the metal substantially altered the resulting barrier height. The barrier heights were determined from the variation of the differential capacitance of the space charge region with applied bias. Crowell et al. ${ }^{1}$ demonstrated that photoresponse measurements of the same structures gave barriers which were compatible with those deduced from capacity measurements although the observed heights seemed to correlate with oxygen-contaminated cases of Archer and Atalla.

The present work reports an experimental investigation of barrier heights from vacuum deposited metals on "cleaved-in-vacuum" samples of $n$-type CdS, $n$-type $\mathrm{GaAs}$, and p-type GaAs. The height of the Schottky barrier was measured by using: (1) the spectral response of the photovoltage, (2) voltage dependence of

${ }^{4}$ R. Williams and R. H. Bube, J. Appl. Phys. 31, 968 (1960).

${ }^{5}$ G. W. Mahlman, Phys. Rev. Letters 7, 408 (1961).

${ }^{6}$ R. J. Archer and M. M. Atalla, Ann. N. Y. Acad. Arts. Sci. 101, 697 (1963).

${ }^{7}$ R. Williams, Phys. Rev. Letters 8, 402 (1962)

${ }^{8}$ C. A. Mead and W. G. Spitzer, Appl. Phys. Letters 2, 74 
the differential capacitance of the space-charge region, and (3) forward biased $I-V$ characteristic of the diode. Some difficulties associated with the interpretation are indicated in addition to those already reported. The results are compared with those of Archer and Atalla and others, and with the conventional model of a surface-barrier rectifier. Some results are interpreted in terms of Fermi level pinning by surface states.

\section{EXPERIMENTAL}

\section{A. Material and Fabrication of Samples}

The CdS sulfide was single-crystal $n$-type material, not purposely doped, and with carrier concentration values ranging from $10^{15}$ to $10^{17} \mathrm{~cm}^{-3}$. The samples were cleaved parallel to the optic axis, which was determined visually with the aid of a polarizing microscope. The $n$-type GaAs samples were all cut from a pulled, Tedoped single crystal grown in the $\langle 111\rangle$ direction. The (110) plane, which is the cleavage plane, was determined in a conventional manner by using an optical goniometer after etching the sample surface with a preferential etch. The free electron concentration, as determined from the Hall coefficient, was $3.8 \times 10^{17} \mathrm{~cm}^{-3}$ at both room temperature and $77^{\circ} \mathrm{K}$. The $p$-type GaAs samples were obtained from a $\mathrm{Zn}$-doped single crystal with a hole concentration of $4.8 \times 10^{16} \mathrm{~cm}^{-3}$ at room temperature and $5.0 \times 10^{15} \mathrm{~cm}^{-3}$ at $77^{\circ} \mathrm{K}$.

Devices were fabricated from small bars of singlecrystal material approximately $2 \times 2 \mathrm{~mm}$ in cross section. The samples were notched and then cleaved in the vacuum system with a small wedge which was struck with a magnetically operated hammer. The vacuum system consisted of an oil-diffusion pump, water-cooled chevron baffle, and an antimigration trap employing Linde $13 \mathrm{x}$ zeolites. Before evaporation the background pressure was typically $1 \times 10^{-7}$ Torr and the pressure rose by a factor of between 2 and 10 during evaporation depending on the metal being evaporated. Evaporation of the metal was commenced before the crystal was cleaved in order to eliminate contamination of the crystal surface by residual gasses. Upon removal from the vacuum system, the cleaved surface was examined under a microscope, and usually consisted of several flat areas separated by multiple-cleavage steps and damaged areas. The flat areas were isolated electrically by flaking off a small amount of the crystal on all sides. Contact was made by a pointed $0.13-\mathrm{mm}$-diam goldwire probe. All units were checked on a $I-V$ curve tracer to display the rectification characteristic.

Prior to cleaving, Ohmic contacts were made to the ends of the bars. The contacts were made on the CdS by cleaving a small section near the end of the bar in air and immediately soldering with indium. Contacts to the $n$ - and $p$-type GaAs were made by soldering a freshly abraded surface with indium doped with Te or $\mathrm{Zn}$, respectively. In the case of GaAs occasional highresistance contacts were encountered. Therefore, wires were soldered on both ends of the bar and the unit checked in the $I-V$ tracer. Only those showing very low impedance were processed further.

\section{B. Methods of Measurement and Interpretation}

The postulated energy-level diagram for a surfacebarrier rectifier has been given a number of times in the literature and is not reproduced here. In the usual model the height of the potential barrier $\varphi_{B}$, measured with respect to the Fermi level is given by

$$
\varphi_{B}=\varphi_{M}-\chi-\Delta_{0}
$$

where $\varphi_{M}$ is the work function of the metal film, $\chi$ is the electron affinity of the semiconductor, and $\Delta_{0}$ is the potential drop across the metal-semiconductor spacing at the interface. It is almost certain that in the many experiments employing chemically prepared semiconductor surfaces the contact between the semiconductor and metal is not an intimate one. Archer and Atalla have pointed out that even for an intimate contact, the work functions would not necessarily be the same as the vacuum values because of changes in the surface-dipole contributions. In addition, Rose ${ }^{9}$ has considered the variations introduced by the different positions that the first metal atoms can occupy with respect to the semiconductor surface. It is also known that if there exists a large concentration of surface states at the semiconductor-metal interface, the interior of the semiconductor becomes screened from the metal ${ }^{10}$ and the height of the potential becomes independent of $\varphi_{M}$. This point is considered further in the next section.

It is of interest to consider each of the techniques employed here to obtain quantitative information on the barrier height.

\section{The Spectral Dependence of the Photoresponse}

Photomeasurements were made on a Gaertner model L234 quartz monochromator and focused-tungsten source. Calibration reference was a Reeder vacuum thermocouple. For photomeasurements the light was chopped at $50 \mathrm{cps}$ at the entrance slit and the photovoltage was amplified by a narrow-band amplifier with 4-MS input impedance and synchronously detected. The light from the exit slit was directly incident on the metalized side of the sample (front wall cell configuration). All photomeasurements were made with the sample at room temperature.

To eliminate all possibility of difficulty due to scattered light, all data used to determine barrier heights were obtained with a 2 -mm-thick GaAs filter in front of the entrance which effectively removed all radiation of wavelength shorter than $\sim 0.95 \mu$. Comparison runs made on typical samples with and without the GaAs filter gave essentially identical barrier heights.

\footnotetext{
${ }^{\circ}$ A. Rose, Concepts in Photoconductivity and Allied Problems (John Wiley \& Sons, Inc., New York, to be published).

${ }_{10}$ J. Bardeen, Phys. Rev. 71, 717 (1949).
} 
As discussed previously, when measuring the photoresponse for $h \nu<E_{g}$, the response per absorbed photon in the metal film is the quantity of interest. However, it was demonstrated that the fraction of the incident energy absorbed by most metals is approximately independent of wavelength for the spectral region of interest in the present work.

The form of the photoresponse has been considered by Crowell et al., ${ }^{1}$ and it is concluded that if $\alpha L>1$ and $\alpha t>1$, where $\alpha$ is the absorption coefficient of the metal, $L$ the electron attenuation length, and $t$ the metal thickness, then the photoresponse has the approximate form

$$
R=C \cdot \alpha \int_{0}^{h \nu-\varphi B} \frac{(\Delta E) e^{-t / L}}{-1 / L+\alpha} d(\Delta E) .
$$

The spectral dependence of $R$ depends upon the energy dependence of $L$. If $L \gg t$, then the familiar Fowler type of dependence is obtained

$$
R \propto\left(h \nu-\varphi_{B}\right)^{2} .
$$

Quinn ${ }^{11}$ has theoretically estimated the energy dependence of the electron mean free path for electronelectron scattering in a metal and concludes that

$$
l=K \frac{1+\left(\varphi_{B}+\Delta E\right) / E_{0}}{\left(\varphi_{B}+\Delta E\right)^{2}},
$$

where $E_{0}$ is the Fermi energy and $\Delta E$ is the excess energy of the electron over the top of the barrier.

Recent Monte-Carlo calculations ${ }^{12}$ of $l$ starting from published values of $L$ indicate that for the metal-film thicknesses and photon energy range considered here $(\sim 1 \mathrm{eV}), l$ and $L$ can have quite different values.

In the present work, it was occasionally necessary to attempt measurements of barriers where $\varphi_{B} \leqslant 0.4 \mathrm{eV}$. In these cases, the photoresponse is weak and it is necessary to make the measurements at photon energies in the range $h \nu=0.6$ to $1.2 \mathrm{eV}$ which is substantially larger than $\varphi_{B}$. If $t \geqslant L$, then attempts to extrapolate the data according to $R^{\frac{1}{2}} \propto h \nu-\varphi_{B}$ can lead to a substantial underestimation of $\varphi_{B}$ because of the energy dependence of $L$. However, if this difficulty is present, then the data is concave towards the photon energy axis. An example of such a curve can be seen in Fig. 1 for the case of $\mathrm{Au}$ on p-type GaAs. The shape of the curve is in general agreement with the energy dependence given by Quinn and the previously reported values of the electron range in gold, but uncertainty as to the details of the transport process and in particular the role of phonon scattering makes exact correlation difficult.

${ }^{11}$ John J. Quinn, Phys. Rev. 128, 1453 (1962).

12 R. N. Stuart, F. Wooten, and W. E. Spicer, Phys. Rev. Letters 10, 7 (1963); F. Wooten, R. N. Stuart, and W. E. Spicer, Bull. Am. Phys. Soc. 8, 254 (1963).

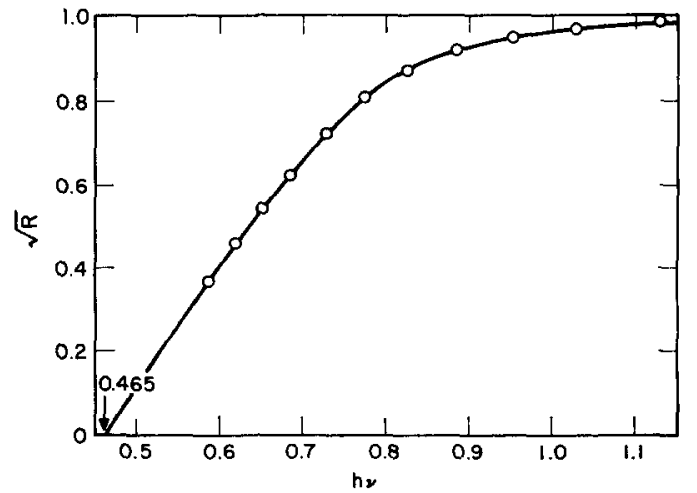

FIG. 1. Photoresponse per incident photon of $A u$ on $p$-type GaAs. Vertical scale in arbitrary units.

\section{Differential Capacitance Measurements}

In this measurement, the change in potential energy in crossing the space-charge region $V_{0}$ is obtained from the $1 / C^{2}=0$ intercept of a $1 / C^{2}$ vs $V$ plot, where $C$ is the space-charge capacity and $V$ is the applied dc reverse bias voltage. The dependence of $C$ on $V$ was determined on a modified Boonton model $74 \mathrm{C}-S 8$ capacitance bridge. The bridge operating frequency was $100 \mathrm{kc}$ and the applied ac voltage was less than $25 \mathrm{mV}$. In those cases where measurements were made at $77^{\circ} \mathrm{K}$, the sample was inserted directly into liquid nitrogen immediately after the room-temperature data had been taken, without breaking contact to the sample.

In order to obtain the barrier height $\varphi_{B}$, it is necessary to add $\left(E_{c}-E_{F}\right)$ or $\left(E_{F}-E_{v}\right)$ to $V_{0}$ depending upon whether the semiconductor bands bend up or down at the interface. $E_{c}, E_{v}$, and $E_{F}$ are the conduction band edge, valence band edge, and Fermi energies in the bulk semiconductor. The $E_{c}-E_{F}$ (or $E_{F}-E_{v}$ ) values are obtained from the carrier concentration ${ }^{13}$ and the relation

$$
R_{H}= \pm 1 / n e,
$$

where $R_{H}$ is the Hall coefficient. Published values of the density of states effective masses, ${ }^{14} m_{d}{ }^{*}$, were used.

Goodman ${ }^{15}$ has recently considered the assumptions which are made in relating the intercept of the capacitance plot to the height of the Schottky barrier. The parameters one reads from the bridge circuit and their relation to the actual device-equivalent circuit, carriertrapping effects, variation of effective surface area with depletion layer width, and minority-carrier concentration within the space-charge region arising from inversion layers were all considered in the light of Goodman's

${ }^{13}$ See, e.g., W. Shockley Electrons and Holes in Semiconductors (D. Van Nostrand, Inc., Princeton, New Jersey, 1950), p. 242. ${ }_{14}$ H. Ehrenreich, J. Appl. Phys. Suppl, 32, 2155 (1961); E. D. Palik, S. Teitler, and R. F. Wallace, J. Appl. Phys. Suppl. 32, 2133 (1961); C. Hilsum and A. C. Rose-Innes, Semiconducting III-V Compounds (Pergamon Press, Inc., New York, 1961), p. 62; J. J. Hopfield and D. G. Thomas Phys. Rev. 122, 35 (1961).

${ }_{15}$ A. M. Goodman, J. Appl. Phys. 34, 329 (1963). 
treatment, and with one exception some simple arithmetic calculations indicated that these effects should cause little difficulty in the present measurements, i.e., the errors introduced being $\leqslant 0.01 \mathrm{eV}$ or less. The exception noted above is the effect of trapping in the $\mathrm{CdS}$ measurements. However, if the diode is biased in the forward direction to flood the electron traps prior to making the capacitance measurements, and if the sample is protected from light then, as described by Goodman, the $1 / C^{2}$ vs $V$ plots are linear and quite reproducible at low-reverse bias $(\leqslant 1 \mathrm{~V})$. Under these conditions, the drift in $C$ was never more than $2 \%$ and in most cases was much less. It is of interest to note that the treatment of all of the above effects predict that the most reliable data are those obtained in the forward bias condition or at small reverse bias.

An effect, not discussed by Goodman, occurs when the metal layer is very thin. Under relatively highreverse bias conditions, the leakage current can become appreciable, and this current flowing through the edgeon spreading resistance of the metal layer causes portions of the metal area far from the contact probe to be less reverse biased than those near the contact. The net effect is a capacitance which changes less rapidly with voltage than expected. Since there is no voltage drop in the absence of applied bias, the zero-bias capacitance should be quite accurate. Hence the indicated value of the barrier height is larger than the true barrier height. Under suitable conditions the $1 / C^{2}$ vs $V$ plot can still approximate a straight line, and it is difficult to determine how much the result has been

TABLE I. A summary of CdS photovoltaic and capacity data; all energies are in $\mathrm{eV} . \Delta E$ is the energy difference in the CdS crystal between the conduction band edge and the Fermi energy. Values of $\Delta E$ followed by $(\rho)$ or $(H)$ were determined from resistivity or Hall measurements. Other values of $\Delta E$ were estimated from $1 / C^{2}$ vs $V$ plots.

\begin{tabular}{|c|c|c|c|c|}
\hline Metal & $\begin{array}{l}\text { Photo- } \\
\text { barrier }\end{array}$ & $\begin{array}{l}V_{0}=1 / C^{2} \\
\text { in tercept }\end{array}$ & $\begin{array}{c}\Delta E \\
\left(E_{c}-E_{H}\right)\end{array}$ & $\begin{array}{c}1 / C^{2} \\
\text { barrier }\end{array}$ \\
\hline \multirow[t]{9}{*}{$\mathrm{Au}$} & $0.75 \pm 0.01$ & $\because$ & $\cdots$ & 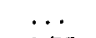 \\
\hline & 0.75 & 0.66 & 0.09 & 0.75 \\
\hline & 0.80 & 0.79 & 0.08 & 0.87 \\
\hline & 0.78 & 0.75 & 0.10 & 0.85 \\
\hline & 0.77 & $\ldots$ & $\ldots$ & $\cdots$ \\
\hline & 0.78 & 0.65 & $0.12(H)$ & 0.77 \\
\hline & 0.79 & $\ldots$ & $\ldots$ & $\ldots$ \\
\hline & 0.78 & $\cdots$ & .. & $\cdots$ \\
\hline & 0.75 & 0.60 & $0.16(\rho)$ & 0.76 \\
\hline \multirow[t]{3}{*}{$\mathrm{Cu}$} & $0.36 \pm 0.02$ & 0.32 & 0.05 & 0.37 \\
\hline & & 0.20 & $0.12(H)$ & 0.32 \\
\hline & 0.36 & 0.30 & $\ldots$ & $\cdots$ \\
\hline \multirow[t]{2}{*}{$\mathrm{Ni}$} & $\sim 0.4-0.5$ & 0.38 & $0.16(\rho)$ & 0.54 \\
\hline & .. & $0.30\left(77^{\circ} \mathrm{K}\right)$ & $\ldots$ & \\
\hline Mo & $\ldots$ & $0.50\left(77^{\circ} \mathrm{K}\right)$ & $\cdots$ & $\cdots$ \\
\hline $\mathrm{Al}$ & & Ohmic cor & & \\
\hline \multirow[t]{3}{*}{$\mathrm{Ag}$} & $0.54(5)$ & 0.45 & $0.16(\rho)$ & 0.61 \\
\hline & 0.55 & 0.40 & $0.16(\rho)$ & 0.56 \\
\hline & 0.58 & $\ldots$ & $\ldots$ & $\cdots$ \\
\hline \multirow[t]{3}{*}{$\mathrm{Pt}$} & 0.84 & 0.70 & $0.16(\rho)$ & 0.86 \\
\hline & 0.82 & $\cdots$ & $\ddot{0}$ & $\ddot{0}$ \\
\hline & $\ddot{0.88}$ & $\begin{array}{l}0.71 \\
0.68\end{array}$ & $\begin{array}{l}0.10(\rho) \\
0.16(\rho)\end{array}$ & 0.84 \\
\hline
\end{tabular}

affected. For this reason samples which showed high forward resistances (few hundred ohms) and relatively high-leakage currents $(\gtrsim 0.1 \mathrm{~mA}$ at $1 \mathrm{~V})$ on the $I-V$ curve tracer were not used for capacitance measurements. The above consideration is particularly important for a system in which $\varphi_{B}$ is small, $\leqslant 0.5 \mathrm{eV}$, and for metals where $L$ is short as in the cases of $\mathrm{Cu}$ and $\mathrm{Al}$. Because of the low photosensitivity and the desire for $t<L$ in order to obtain the simple Fowler plot, it is reasonable to prepare samples with thin metal films, of the order of $100 \AA \AA$. Therefore, in such cases, it can be observed that photoresponse and capacity data are not necessarily taken on the same sample.

Since, in the present work, the sample is cleaved in the stream of the evaporating metal in the vacuum system, contamination of the interface is effectively eliminated. Where a surface layer is present Goodman has shown that under suitable conditions

$V_{0}=\varphi_{B}-\left(E_{c}-E_{F}\right)+\left[n e \epsilon t^{2} / 2 \epsilon_{i}^{2}\right]+\left[2 e \epsilon n V_{0}\right]^{\frac{1}{2}} t / \epsilon_{t}$,

where $\epsilon$ is the semiconductor dielectric constant, $t$ the effective thickness of the surface layer, and $\epsilon_{t}$ the dielectric constant of the surface layer. In the measurements which use $n$-type GaAs the correction terms (the last two terms in the above equation) may be appreciable depending upon the values of $t$ and $\epsilon_{t}$. For the $p$-type GaAs and the CdS the carrier concentrations are reduced by an order of magnitude or more and the correction terms are $\sim 0.01 \mathrm{eV}$ or less.

\section{Diode Forward Characteristic Measurements}

The $I-V$ characteristic in the forward direction where $V>$ few tenths of a volt is of the form $I=I_{0} \exp$ $(e V / a k T)$, where $a \geqslant 1$. The plot of $\log I$ vs $V$ is extrapolated to $V=0$ and the $\varphi_{B}$ deduced from $I_{0}$ and the Richardson emission equation. There is considerable difficulty in obtaining any better than order of magnitude accuracy in $I_{0}$ even at room temperature. At forward currents greater than $1-10 \mathrm{~mA}$ the series resistance coming from the bulk semiconductor and, in some cases, the spreading resistance of the metal film. start to limit the current, and for $I \leqslant 10 \mu \mathrm{A}$ the contribution from leakage is of ten important. Therefore, the $\varphi_{B}$ from this measurement was only checked to see if reasonable (within $\sim 0.1 \mathrm{eV}$ ) agreement was obtained with the $\varphi_{B}$ from the other methods. In almost all cases, such agreement was obtained at room temperature. At lower temperatures the $\log I$ vs $V$ curve for GaAs shifted to larger voltages but the slope did not indicate an appreciable change even at $77^{\circ} \mathrm{K}$. At the present time this behavior is not understood and casts doubt on the $\varphi_{B}$ obtained by this procedure.

\section{EXPERIMENTAL RESULTS AND DISCUSSION}

\section{A. Cadmium Sulfide}

Table I summarizes the results of the present measurements on $n$-type CdS. The measurements for $\mathrm{Au}$ and 
TABLE II. A summary of CdS photovoltaic and capacity data for samples processed as indicated.

\begin{tabular}{|c|c|c|c|c|c|}
\hline Metal & Process & Photobarrier & $1 / C^{2}$ intercept & $\Delta E$ & $1 / C^{2}$ barrier \\
\hline \multirow[t]{4}{*}{$\mathrm{Au}$} & Cleave and etch & $0.82 \pm 0.02$ & $0.76 \pm 0.01$ & 0.10 & 0.86 \\
\hline & $\begin{array}{l}\text { Cleaved } \\
\text { Cleaved }\end{array}$ & $1.15 \pm 0.02$ & $\approx \begin{array}{l}4.5 \\
105\end{array}$ & $\ddot{0 i 7}$ & iㅜ \\
\hline & Cleaved & $\cdots$ & Over 2.0 & $\begin{array}{l}0.11 \\
\cdots\end{array}$ & $\begin{array}{l}1.22 \\
\ldots\end{array}$ \\
\hline & Cleave and etch & .. & $\approx 2.5$ & $\cdots$ & $\cdots$ \\
\hline \multirow[b]{3}{*}{$\mathrm{Cu}$} & Lapped and etched & $0.88 \pm 0.33$ & $\approx 2.5$ & $\ldots$ & $\ldots$ \\
\hline & Cleaved & $0.82 \pm 0.05$ & $0.70 \pm 0.02$ & 0.17 & 0.87 \\
\hline & Cleaved & $0.60 \pm 0.01$ & $\begin{array}{l}\left(0.5 \neq 0.3-1 / C^{-2}\right. \\
\text { vs } V \text { not straight line })\end{array}$ & & $\cdots$ \\
\hline
\end{tabular}

$\mathrm{Cu}$ have been indicated in a previous paper. ${ }^{8}$ The $\Delta E(H)$ was calculated from Hall measurements as described previously with $m_{d}{ }^{*}=0.5 m_{0}$. The $\Delta E(\rho)$ was estimated from resistivity data assuming the electron mobility, ${ }^{16} \mu_{e}=250 \mathrm{~cm}^{2} / \mathrm{V} \mathrm{sec}$. The other values of $\Delta E$ were obtained from the slope of the $1 / C^{2}$ vs $V$ plot and the area of contact. As discussed in a previous paper, ${ }^{8}$ the latter method can be inaccurate, however, in some cases it was the only practical measurement.

Comparison of the photobarrier values with those obtained from capacity measurements clearly show the necessity of taking the Fermi energy into account. The agreement between the two types of $\varphi_{B}$ measurements, except for a few isolated cases, is as good as the agreement among the various values obtained for a single metal from either type of measurement, i.e., a few hundredths of a volt. The barrier height shows a strong dependence upon the particular metal used ranging from $0.85 \pm 0.03 \mathrm{eV}$ for Pt to an Ohmic contact $\left(\varphi_{B}<0.10 \mathrm{eV}\right)$ for Al. In changing the metal work function by $\sim 1.1 \mathrm{v}$ the barrier height changes by at least $0.75 \mathrm{~V}$. In view of our previous remarks, there exists an almost surprisingly good relation between the two quantities. It should be noted, however, that there are other quantities which show a strong empirical relation to $\varphi_{B}$. For example, an even better correlation exists between $\varphi_{B}$ and the electronegativity values given by Pauling ${ }^{17}$ and suggests a possible role played by the partially ionic nature of the semiconductor-metal bond in determining the value of $\varphi_{B}$.

Several conclusions can be drawn from the CdS data. It has been proposed ${ }^{18,19}$ that the photoresponse for $h \nu<E_{0}$ is due to impurity excitation in the CdS, or the formation of a $p-n$ junction with excitation from the impurity levels (i.e., the $\mathrm{Cu} 3 d$ level) in the $p$ region. In a recent letter ${ }^{8}$ the present authors pointed out that on the basis of the $\mathrm{Au}$ and $\mathrm{Cu}$ results neither explanation would suffice to explain the values of $\varphi_{B}$ for vacuum cleaved samples. The complete list of data given in Table I substantiates this latter viewpoint. If the photo-

\footnotetext{
${ }^{16}$ W. W. Piper and D. T. F. Marple, J. Appl. Phys. 34, 2237 (1963).

${ }_{17}$ L. Pauling, The Nalure of the Chemical Bond (Cornell University Press, Ithaca, New York, 1960), Chap. 3.

${ }_{18}$ E. D. Fabricius, J. Appl. Phys. 33, 1597 (1962).

${ }^{19}$ H. G. Grimmeiss and R. Memming, J. Appl. Phys. 33, 2217 (1962).
}

response were due to some impurity present in the CdS prior to evaporation of the metal film it would be difficult to explain the systematic variation in $\varphi_{B}$ nor would there be any a priori reason for the agreement of the values of $\varphi_{B}$ from. the two types of measurements. If the response were due to impurities in the metal evaporated, which is very unlikely with the purity material used, then in addition to the above objections there would be no reason for any correlation between $\varphi_{B}$ and the metal work function.

The above discussion does not, however, apply to the CdS-metal system when the cleaved surface has been exposed to the atmosphere prior to the evaporation of the metal film. The data for a number of samples in which the CdS surface was prepared as indicated are given in Table II. In the present case, elaborate precautions were not taken to insure reproducibility of atmospheric conditions, time of exposure, purity of etching solution, etc. It is apparent that the results are much less reproducible. In some cases, $\varphi_{B}$ is similar to the vacuum-cleaved samples. In other cases, the two measurements of $\varphi_{B}$ give different results, and often the $1 / C^{2}$ vs $V$ data predict very large barriers. In the light of these measurements difficulties in comparing data obtained by different investigators employing different techniques of sample preparation becomes apparent.

Goodman ${ }^{15}$ has published $V_{0}$ values for some $\mathrm{Au}-\mathrm{CdS}$ samples. The $\mathrm{CdS}$ was etched $(6 M \mathrm{HCl})$ and the $\mathrm{Au}$ was electroplated. The values of $\varphi_{B}$ deduced from capacity measurements for three cases are $0.93,1.08$, and $0.93 \mathrm{eV}$. These values are all larger than the largest value obtained on the $\mathrm{Au}-\mathrm{CdS}$ vacuum-cleaved samples but within the range of $\varphi_{B}$ for the other samples which gave "reasonable" results, i.e., eliminating those which gave barriers of several volts and probably involve some type of interfacial dielectric layer. In more recent work Goodman ${ }^{20}$ has reported a $\varphi_{B}=0.68 \mathrm{eV}$ for Au evaporated on an etched surface.

The results obtained here may also be compared to the earlier work of Williams and Bube ${ }^{4}$ in which the $\mathrm{Cu}-\mathrm{CdS}$ system gave $\varphi_{B}=1.1 \mathrm{eV}$ from photoresponse measurements while some experiments on the quantum yield of photocurrent as a function of the $\mathrm{CdS}$ conductivity indicated a $\varphi_{B} \sim 0.4 \mathrm{eV}$. The $\varphi_{B}$ can be estimated

${ }^{20}$ A. M. Goodman, Bull. Am. Phys. Soc. 8, 210 (1963). 


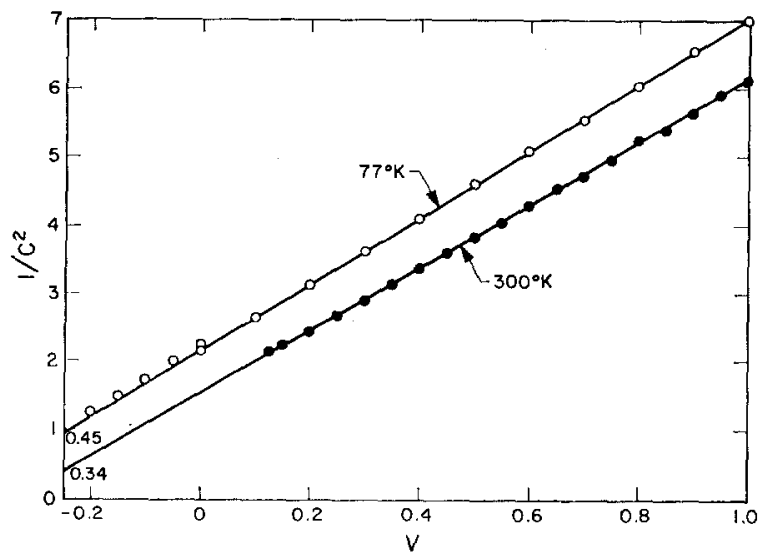

FIG. 2. Capacitance of Au on p-type GaAs (same sample as Fig. 1). Vertical scale in arbitrary units but same for both curves.

from the forward diode characteristic given in Fig. 3 of this same paper and is $\sim 0.6-0.7 \mathrm{eV}$. Again, these films were electroplated so comparison to the present measurements is difficult. It is of interest to note that the $\sim 0.4 \mathrm{eV}$ is close to the $\varphi_{B}$ measured here, however, in view of the photoresponse and diode values, this agreement is probably accidental.

\section{B. Gallium Arsenide}

Tables III and IV summarize the results of the measurements of the $n$-type and $p$-type GaAs units, respectively. The $E_{c}-E_{F}$ and $E_{F}-E_{v}$ values were determined by room temperature and liquid nitrogen Hall coefficient measurements. Figures $4,5,6$, and 7 show photoresponse and capacitance plots for $\mathrm{Al}$ samples. It is noted that the room temperature and $77^{\circ} \mathrm{K}$ plots of $1 / C^{2}$ vs $V$ have nearly the same slopes. This result was expected for the $n$-type sample since according to simple theory the slope is given by

$$
d\left(1 / C^{2}\right) / d V=2 / q N_{D} \in A^{2},
$$

where $N_{D}$ is the ionized donor concentration, $A$ the area of contact, and $\epsilon$ the semiconductor dielectric constant. Hall-coefficient measurements at the two temperatures

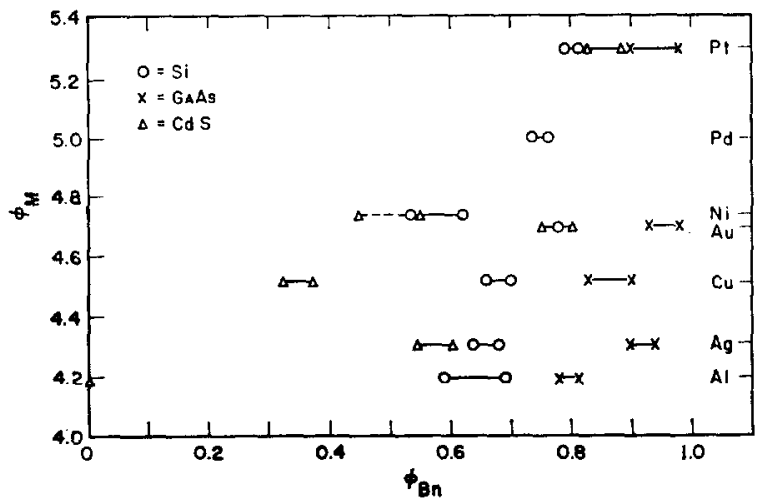

FIG. 3. A comparison of barrier height s obtained for various metals on $n$-type CdS, Si, and GaAs.
TABLE III. Photo- and capacity-barrier heights obtained on vacuum-cleaved $n$-type GaAs samples. For all samples $E_{c}-E_{F}=0$ at room temperature and $=-0.03 \mathrm{eV}$ at $77^{\circ} \mathrm{K}$. Values for $77^{\circ} \mathrm{K}$ immediately follow room-temperature values for the same samples.

\begin{tabular}{ccc}
\hline Metal & Photobarrier & $1 / C^{2}$ barrier \\
\hline $\mathrm{Au}$ & $\ldots$ & 0.93 \\
& $\ldots .90$ & 0.95 \\
$\mathrm{Pt}$ & 0.88 & 0.98 \\
& $\ldots .86$ & 0.98 \\
& 0.84 & $\ldots .93$ \\
& 0.88 & 0.90 \\
$\mathrm{Be}$ & 0.82 & 0.90 \\
& 0.81 & 0.82 \\
$\mathrm{Ag}$ & $\ldots$ & 0.95 \\
& $\ldots$ & 0.90 \\
& 0.88 & 0.94 \\
$\mathrm{Cu}$ & 0.89 & 0.94 \\
& 0.78 & 0.83 \\
& 0.76 & $\ldots$ \\
& 0.82 & 0.90 \\
& $\ldots$ & 0.85 \\
& $\ldots .88$ & $\ldots$ \\
$\mathrm{Sn}$ & 0.83 & 0.68 \\
& 0.67 & 0.74 \\
& 0.63 & 0.73 \\
$\mathrm{Ba}$ & $\ldots$ & 0.68 \\
$\mathrm{Al}$ & $\ldots .94$ \\
& $\ldots .80$ & 0.81 \\
& $\ldots$ & $0.92\left(77^{\circ}\right)$ \\
& $\ldots .78$ \\
& $\ldots .85$ & 0.80 \\
& $\left.\ldots .77^{\circ}\right)$ \\
& $\ldots .85\left(77^{\circ}\right)$ \\
\hline
\end{tabular}

showed no change in $N_{D}$. It may also be remarked that the concentration of compensating acceptor levels $N_{A}$ is an order-of-magnitude less than $N_{D}$ for these samples. For the p-type sample the bulk ionized acceptor concentration (assumed equal to the hole concentration) decreases by approximately one order of magnitude between room temperature and $77^{\circ} \mathrm{K}$. The slight change in slope of the $1 / C^{2}$ curve indicates only a small change in ionized acceptor concentration in the space-charge region.

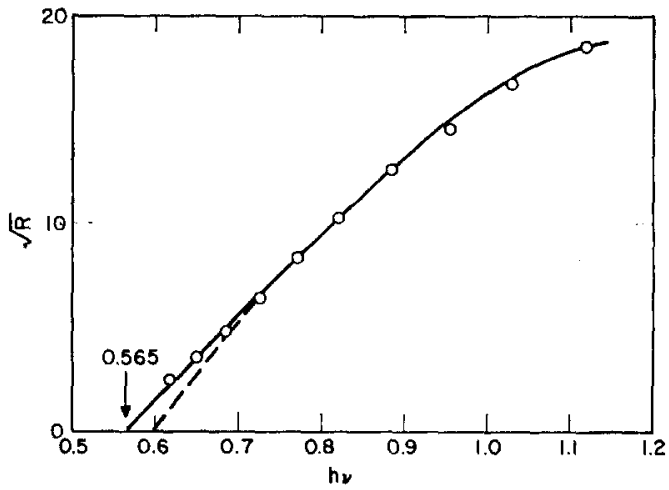

Fig. 4. Photoresponse of typical Al on p-type GaAs sample. Vertical scale arbitrary. 
BARRIER HEIGHTS STUDIES ON METAL-SEMICONDUCTOR SYSTEMS 3067

TABLE IV. Photo- and capacity-barrier heights obtained on vacuum cleaved, $p$-type GaAs samples. For all samples $E_{F}-E_{v}$ $=0.13 \mathrm{eV}$ at room temperature $=0.04 \mathrm{eV}$ at $77^{\circ} \mathrm{K}$. Values for $77^{\circ} \mathrm{K}$ immediately follow room-temperature values for the same samples.

\begin{tabular}{|c|c|c|c|}
\hline Metal & $\begin{array}{l}\text { Photo- } \\
\text { barrier }\end{array}$ & $\begin{array}{c}1 / C^{2} \\
\text { intercept }\end{array}$ & $\begin{array}{c}1 / C^{2} \\
\text { barrier }\end{array}$ \\
\hline \multirow[t]{5}{*}{$\mathrm{Au}$} & 0.46 & 0.34 & 0.47 \\
\hline & $\cdots$ & 0.45 & 0.49 \\
\hline & $\sim 0.38$ & $\cdots$ & $\ldots$ \\
\hline & $\ldots$ & 0.42 & $0.46\left(77^{\circ}\right)$ \\
\hline & $\ldots$ & 0.42 & $0.46\left(77^{\circ}\right)$ \\
\hline \multirow[t]{2}{*}{$\mathrm{Pt}$} & $\cdots$ & 0.44 & $0.48\left(77^{\circ}\right)$ \\
\hline & $\ldots$ & 0.44 & $0.48\left(77^{\circ}\right)$ \\
\hline \multirow[t]{2}{*}{$\mathrm{Be}$} & $\sim 0.3$ & & $\ddot{\cdots}$ \\
\hline & $\ldots$ & 0.37 & $0.41\left(77^{\circ}\right)$ \\
\hline $\mathrm{Ag}$ & $\cdots$ & 0.40 & $0.44\left(77^{\circ}\right)$ \\
\hline \multirow{4}{*}{$\mathrm{Cu}$} & $\cdots$ & 0.48 & $0.52\left(77^{\circ}\right)$ \\
\hline & $\ldots$ & 0.46 & $0.50\left(77^{\circ}\right)$ \\
\hline & $\ldots$ & 0.48 & $0.52\left(77^{\circ}\right)$ \\
\hline & $\cdots$ & 0.49 & $0.53\left(77^{\circ}\right)$ \\
\hline \multirow[t]{6}{*}{$\mathrm{Sn}$} & 0.55 & $\cdots$ & $\ldots$ \\
\hline & $\cdots$ & 0.58 & 0.71 \\
\hline & $\ldots$ & 0.69 & $0.73\left(77^{\circ}\right)$ \\
\hline & $\cdots$ & 0.58 & 0.71 \\
\hline & $\cdots$ & 0.52 & 0.65 \\
\hline & $\cdots$ & 0.58 & $0.62\left(77^{\circ}\right)$ \\
\hline \multirow{12}{*}{$\mathrm{Al}$} & $\ldots$ & 0.50 & 0.63 \\
\hline & $\ldots$ & 0.61 & $0.65\left(77^{\circ}\right)$ \\
\hline & 0.52 & 0.50 & 0.63 \\
\hline & $\cdots$ & 0.57 & $0.61\left(77^{\circ}\right)$ \\
\hline & 0.45 & 0.44 & 0.57 \\
\hline & $\cdots$ & 0.52 & $0.56\left(77^{\circ}\right)$ \\
\hline & 0.44 & 0.47 & 0.60 \\
\hline & & 0.53 & $0.57\left(77^{\circ}\right)$ \\
\hline & 0.54 & 0.52 & 0.65 \\
\hline & $\cdots$ & 0.58 & $0.62\left(77^{\circ}\right)$ \\
\hline & 0.56 & 0.56 & 0.69 \\
\hline & $\cdots$ & 0.61 & $0.65\left(77^{\circ}\right)$ \\
\hline
\end{tabular}

The room-temperature value of $\varphi_{B}$ for all metals, with the exception of $\mathrm{Sn}$, on $n$-type GaAs, is between $\sim 0.80$ and $0.98 \mathrm{eV}$. This is to be contrasted to the strong dependence of $\varphi_{B}$ on $\varphi_{M}$ for the same metals on CdS.

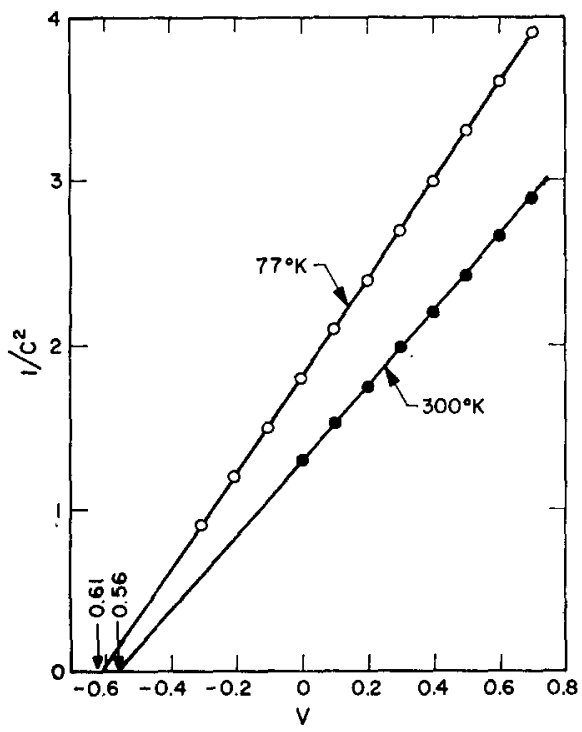

FIG. 5. Capacity data on sample of Fig. 4. Vertical scale arbitrary but same for both curves.

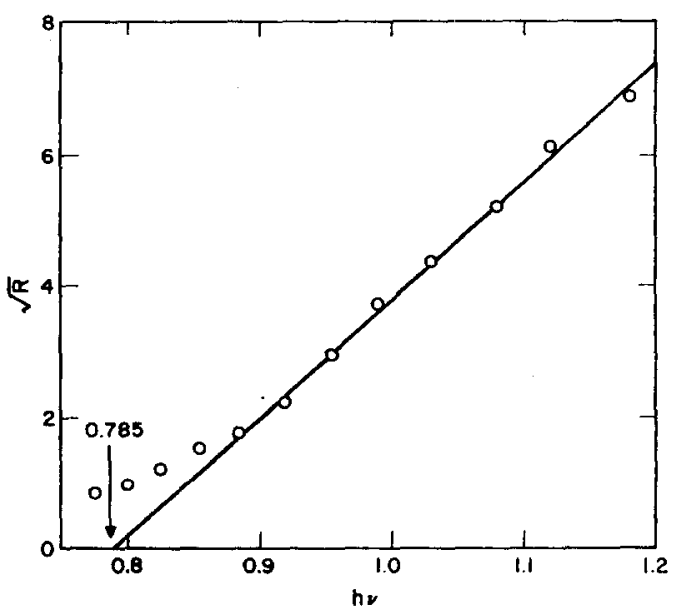

FIG. 6. Photoresponse of typical Al on $n$-type GaAs sample. Vertical scale arbitrary.

The agreement between $\varphi_{B}$ for the two types of measurement is not as good as previously noted for CdS. The room temperature and liquid-nitrogen carrier concentrations for the $n$-type GaAs are $4 \times 10^{17} \mathrm{~cm}^{-3}$ and, as previously indicated, the correction terms in Eq. (5) may be as large as several hundredths of a volt, making $V_{0}+\left(E_{c}-E_{F}\right)$ exceed $\varphi_{B}$ by this amount. It may be noted that $\varphi_{B}$ from photomeasurements does show a tendency to be somewhat less than $\varphi_{B}$ from capacity measurements.

The lack of sensitivity of $\varphi_{B}$ on $\varphi_{M}$ for the $n$-type samples is also observed for the $p$-type samples as

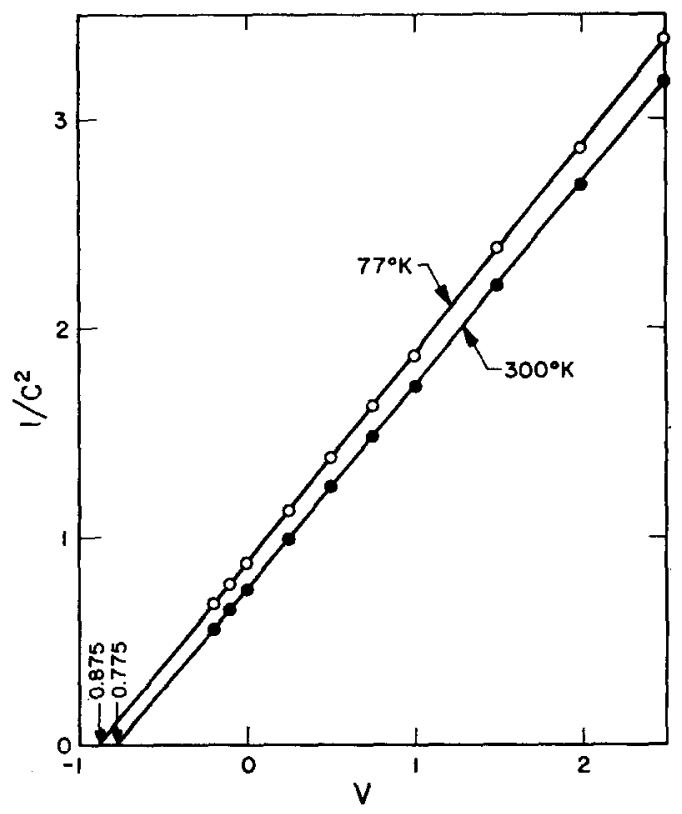

FIg. 7. Capacity data on sample of Fig. 6. Vertical scale arbitrary but same for both curves. 
indicated in Table IV. Because of large leakage current for some materials it was difficult to obtain reliable photodata and good capacity measurements could only be made at low temperature. However, for the cases of $\mathrm{Al}, \mathrm{Au}$, and $\mathrm{Sn}$, the leakage currents were substantially lower, capacity measurements were made both at room temperature and $77^{\circ} \mathrm{K}$, and reliable photodata were obtained. Figures 1 and 2 show photoresponse and capacity data for an Au sample. The photoresponse curve is not a straight line but concave, as previously described. Except for this sample, a major discrepancy is noted in the barriers deduced from the two measurements. The $\varphi_{B}$ (photo) consistently agrees much more closely with $V_{0}$ than with $\varphi_{B}$ (capacity), and the room. temperature value of $E_{F}-E_{v}=0.13 \mathrm{eV}$. The carrier concentration, $p=4.8 \times 10^{16} \mathrm{~cm}^{-3}$, was checked on Hall samples taken from the GaAs crystal immediately above and below the section used for the cleavage samples. Less than $10 \%$ difference was noted in the carrier concentration. At the present time, the authors do not have a satisfactory explanation for this discrepancy. Because of the curvature of the photoresponse curve for Al the correct $V_{0}$ may be uncertain, to at most, $0.05 \mathrm{eV}$. Photoresponse data for thin $\mathrm{Al}$ samples (Al thickness $\sim 200 \AA$ ), where the data lie on a straight line as simple Fowler theory predicts, also indicate the same discrepancy.

Regardless of whether the previously described work function model of a surface barrier rectifier applies or if the Fermi energy is pinned at the interface by a large concentration of surface states, the barriers measured on $n$-type and $p$-type material, $\varphi_{B n}$ and $\varphi_{B p}$, should give

$$
\varphi_{B n}+\varphi_{B p}=E_{q},
$$

where $E_{g}$ is the semiconductor energy gap. This assumes that if surface states are important, they are the same on both the $n$-and $p$-type surfaces when the metal film has been deposited. $\mathrm{Al}$ is the only metal for which we have $\varphi_{B n}$ and $\varphi_{B}$ measurements at both temperatures. $\tilde{\varphi}_{B n}\left(300^{\circ} \mathrm{K}\right)=0.79 \mathrm{eV}$ and $\bar{\varphi}_{B p}\left(300^{\circ} \mathrm{K}\right)=0.63 \mathrm{eV}$ giving $E_{g} \simeq 1.42 \mathrm{eV}$. This result is slightly higher than the values usually given, ${ }^{21} E_{g}=1.35-1.40 \mathrm{eV}$, however, it has already been noted that $\varphi_{B n}$ (capacity) may be a few hundredths of a volt too large. At liquid-nitrogen temperature $\bar{\varphi}_{B n}=0.87 \mathrm{eV}$ and $\bar{\varphi}_{B p}=0.61 \mathrm{eV}$ giving an $E_{g}=1.48 \mathrm{eV}$ compared to $E_{g}=1.46-1.48 \mathrm{eV}$ in the literature. Values of $\varphi_{B} p\left(300^{\circ} \mathrm{K}\right)-\varphi_{B p}\left(77^{\circ} \mathrm{K}\right)$ measured for $\mathrm{Sn}-\mathrm{GaAs}$ samples and given in Table IV are in reasonable agreement with the same quantity measured for the $\mathrm{Al}-\mathrm{GaAs}$ samples. The agreement between the various $E_{g}$ values is regarded as satisfactory, particularly in view of the variability of the $\varphi_{B}$ values between different samples.

${ }^{21}$ T. S. Moss, Optical Properites of Semiconductors (Academic Press Inc., New York, 1959), p. 224; Semiconducling III-V Compounds (Pergamon Press, Inc., New York, 1961).
The similarity of the results obtained here and those previously reported for silicon are shown by Fig. 3 where $\varphi_{M}$ is plotted against $\varphi_{B n}$ for both silicon and GaAs. The silicon data are taken from Archer and Atalla. It is observed that the $\varphi_{B n}$ for the GaAs samples are consistently larger by $0.15-0.30 \mathrm{eV}$ than for the silicon which brackets the difference in the forbidden energy gaps for the two semiconductors. This would indicate that the Fermi level is pinned at the surface at an energy above $E_{v}$ which is nearly the same for each system. Moreover, the fact that $\varphi_{B p}$ shows very little change between $300^{\circ}$ and $77^{\circ} \mathrm{K}$ indicates that the surface states responsible for fixing the Fermi level position remain fixed with respect to the valence band edge.

According to the theory of Bardeen, ${ }^{10}$ at a surfacestate concentration $\gg 10^{13} \mathrm{~cm}^{-2}$ the barrier height becomes insensitive to the metal work function. The results of the $\mathrm{GaAs}$ and the previous work for $\mathrm{Si}$ indicate that this condition is close to being realized for a number of different metal contacts. Because of the techniques employed in making the diodes the surface states are assumed to be in intimate contact with the semiconductor and hence are what is commonly called "fast states".

The only previously available data for GaAs are those of Williams ${ }^{7}$ for Sn on $p$-type material. Again, in this case, the semiconductor surface was etched and the metal film electrodeposited. The $\varphi_{B p}$ was determined from the same measurements used here and values of $0.84 \pm 0.05 \mathrm{eV}$ (capacity), $0.75 \mathrm{eV}$ (photoresponse), and $0.79 \mathrm{eV}\left(I-V\right.$ characteristic) were obtained. The $\varphi_{B p}$ in the present study is significantly lower than the above values; however, it is of interest to note that as in the measurements reported here, the above values have $\varphi_{B p}$ (photoresponse) $<\varphi_{B p}$ (capacity) by $\sim 0.1 \mathrm{eV}$.

The GaAs measurements reported here have not demonstrated that $\varphi_{B n}$ is independent of the position of the bulk Fermi level. Increasing $n=4 \times 10^{17} \mathrm{~cm}^{-3}$ by over an order of magnitude gives units in which the tunnel current can start to be appreciable, and decreasing $n$ by the same amount gives samples in which compensation is important, that is, $N_{\text {Donor }} \sim N_{\text {Acceptor. }}$ Therefore, the total variation of $E_{c}-E_{F} \sim 0.15 \mathrm{eV}$ which is not large compared to the spread in values of $\varphi_{B n}(\sim 0.05 \mathrm{eV})$ for units of a given metal-GaAs system. However, since $\left(E_{c}-E_{F}\right)$ at the surface is very nearly the same for the $n$ - and $p$-type samples, it appears that the assumption $\varphi_{B}$ is independent of $E_{F}$ is reasonable. In the case of the Au-silicon system, Archer and Atalla have shown this assumption to be a valid one.

Early vacuum-photoelectric emission and workfunction data have been reported ${ }^{22}$ for $n$-type GaAs with ground and broken surfaces. The broken surface consisted primarily of (110) regions. The work function reported is $4.69 \mathrm{eV}$ and the energy difference between

${ }^{22}$ D. Haneman and E. W. J. Mitchell, J. Phys. Chem. Solids 15, 82 (1960). 
$E_{F}$ and $E_{v}$ at the surface is $\sim 0.3 \mathrm{eV}$. This value for $\left(E_{F}-E_{v}\right)_{\text {surface }}$ is quite close to the values obtained here considering that the comparison is between a "free" surface and one with a metal film covering it. The more recent work of Gobeli and Allen ${ }^{23}$ on vacuumcleaved GaAs give a minimum-energy separation

${ }^{23}$ G. W. Gobeli and F. G. Allen, Bull, Am. Phys. Soc. 8, 189 (1963).
$\left(E_{F}-E_{v}\right)_{\text {surface }}=0.72 \mathrm{eV}$, a value which clearly does not correspond to the metal-GaAs system.

\section{ACKNOWLEDGMENTS}

The authors wish to express their appreciation to $D$. Reynolds who supplied the CdS crystals, R. Willardson and W. Allred who furnished the GaAs, and H. M. Simpson who fabricated the samples.

\title{
Microplasma Breakdown in Germanium
}

\author{
M. Poleshuk and P. H. Dowling \\ Philips Laboratories, Irvington-on-Hudson, New York \\ (Received 11 April 1963; in final form 27 May 1963)
}

\begin{abstract}
Interpretation of breakdown results in Ge diodes is frequently complicated by effects associated with surface excess current. When these effects are minimized, breakdown is observed within the junction at a "breakdown center," starting at a definite voltage $V_{B}$, and is accompanied by the onset of microplasma pulses. In any one diode, there may be a number of centers, each having its characteristic value of $V_{B}$ and producing characteristic microplasma pulses. The minimum value of $V_{B}$ determines the breakdown voltage of the diode and it is possible to increase the latter radically by etching away centers having lower values of $V_{B}$.

Observations were made at temperatures from $-253^{\circ}$ to $27^{\circ} \mathrm{C}$ on $\mathrm{Ge}$ alloy junctions (n-type base resistivities from 0.54 to $5.4 \Omega-\mathrm{cm}$ ). The properties of the pulses are discussed in some detail: the effect of raising the voltage above $V_{B}$, the effect of light, and the temperature coefficient of $V_{B}$. Values of the last are sufficiently high to suggest that suitable diodes can be used as cryogenic thermometers capable of reading smaller changes than $0.01^{\circ} \mathrm{C}$ at $-253^{\circ} \mathrm{C}$.

Various aspects of the microplasma breakdown are discussed: the mechanism for triggering a pulse and that for "turning it off," the role of the spreading resistance, the possible role of a negative resistance at breakdown, and the effect of microplasma breakdown on the measurement of carrier multiplication at voltages in the vicinity of breakdown.
\end{abstract}

\section{INTRODUCTION}

$T$ HERE has been a considerable amount of work on the breakdown of semiconducting diodes, but most of this has been on silicon diodes at room temperature. Less work has been done on germanium diodes, ${ }^{1-6}$ also mainly at room temperature, but this is frequently marred by a failure to ensure that actual breakdown was being observed rather than the effects of excess surface current ("soft knee," "soft Zener" volt-ampere characteristic, or "surface breakdown").

In a rather extensive series of observations on germanium diodes, we find that when care is taken to minimize the excess surface current, true breakdown within the junction area is observable at room temperature and is indicated by the onset of characteristic pulses which are qualitatively the same as those ob-

\footnotetext{
${ }^{1}$ K. B. Mc $A f e c$, E. J. Ryder, W. Shockley, and M. Sparks, Phys. Rev. 83, 650 (1951).

${ }_{2}^{2}$ K. B. McAfee and K. G. McKay, Phys. Rev. 92, 858 (1953).

${ }^{3}$ S. L. Miller, Phys. Rev. 99, 1234 (1955).

${ }^{4}$ R. D. Knott, I. D. Colson, and M. R. P. Young, Proc. Phys. Soc. (London) B68, 182 (1955).

${ }^{5}$ D. R. Muss and R. F. Greene, J. Appl. Phys. 29, 1534 (1958).

6 T. Tokuyama, Solid-State Electron. 5, 161 (1962).
}

served at the breakdown of silicon diodes. These are, however, of such short duration that they are extremely difficult to resolve. At low temperatures, the excess surface currents present no problem and the pulses at breakdown usually become of longer duration so that their properties can be studied readily.

In Sec. II we describe the experimental diodes and measuring circuit used during this investigation. Characteristic properties of soft-knee and actual junction breakdown are reported in Secs. III and IV, respectively. In Sec. V we demonstrate the presence of microplasma breakdown centers within germanium junctions and discuss the effect of their removal on the breakdown voltage of the diode. Section VI deals with the interpretation of breakdown phenomena in terms of various breakdown criteria. In Sec. VII we discuss breakdown at low temperatures including the pulse properties, pulse-triggering mechanisms, diode volt-ampere characteristics, the possible role of spreading resistance in limiting breakdown current, and the temperature dependence of microplasma breakdown voltage. Application of some of these results to the measurement of cryogenic temperatures with a micropulsing diode is described in Sec. VIII. Section IX is devoted to a dis- 